\title{
Analysis of $M^{x} / G(\mathbf{a}, \mathbf{b}) / 1$ Queueing System with Two Phases of Service Subject to Server Breakdown and Extended Bernoulli Vacations
}

\author{
G. Ayyappan ${ }^{1}$, R. Supraja ${ }^{2}$ \\ ${ }^{I}$ Department of Mathematics, Pondicherry Engineering College, Pillaichavady, Puducherry, India. \\ ${ }^{2}$ Department of Mathematics, Pondicherry Engineering College, Pillaichavady, Puducherry, India. \\ *Corresponding Author: R. Supraja, Department of Mathematics, Pondicherry Engineering College, \\ Pillaichavady, Puducherry, India.
}

\begin{abstract}
This paper deals with a batch arrival queueing system with a single server providing two phases of heterogeneous service to a batch of customers with dissimilar service rates. After attainment of two successive phases of service, the server takes the first type of Bernoulli vacation. At the end of Bernoulli vacation, the server has the option to take the second type of extended vacation. Although the server is functioning with any phase of service, it may breakdown at any moment and the service channel will break off for a short period of time. As a result of breakdown, a batch of customers in either phase of service is interrupted, the service channel sent to repair process immediately. The repair process presumed to be general distribution. We derive the probability generating function of the number of customers in the queue at a random epoch in transient and steady state conditions and also we obtained the queue size distribution at a departure epoch under the steady state conditions. Performance measures in the different states of the system, the mean queue size and the average waiting time in the queue are obtained explicitly. Some particular cases are determined. A numerical illustration is given.
\end{abstract}

Keywords: Bulk service; Bernoulli vacation; Optional vacation; Random breakdown; Repair time.

AMS Subject Classification (2010) 60K25, 90B22, 68M20

\section{INTRODUCTION}

Batch arrival and bulk service queueing systems are quite common in many real life situations such as the arrival of aircraft passengers, elevators, Manufacturing systems, communication network, giant wheel, tourism, etc. Bulk service queueing model was initially originated with Bailey [1]. Neuts [2] proposed the "General Bulk Service Rule" in which service initiates only when a certain number of customers in the queue is available. Some general bulk service results have discussed by Holman, Chaudhry, and Ghosal [3]. Chaudhry and Templeton [4] have worked on bulk service rule by using the supplementary variable technique. Banerjee et.al [5] has considered a queueing model with variable service capacity and batch-size-dependent service. Sikdar and Samanta [6] have analyzed a finite buffer queueing model with bulk service variable server capacity. Recently, bulk service queueing model with multiple working vacations have studied by Jeyakumar and Senthilnathan [7].

Two phase queueing system with Bernoulli vacation using the supplementary variable method studied by many researchers such as Choudhury and Deka [8] and Badamchi Zadeh and Shahkar [9]. Recently, bulk service retrial queueing system has examined by Haridass et.al [10]. Bulk service queueing model with vacations is useful to do some kind of job is dealing with server vacation. Bernoulli vacation with two phases of single service considered by several authors such as Choudhury [11] and Madan [12]. Ayyappan and Shymala [13] have studied a batch arrival single server system with second optional server vacation. Bulk service queueing model with Bernoulli vacation have considered by Al-Khedhairi and Tadj [14].

In all the queueing literature presume that the server is active in the service station at all time. However, these assumptions are impractical. In a practical system, we frequently faced the case where the service station may break off and can be repaired immediately. Many researchers such as 
Rajadurai et.al [15], Choudhury and Deka [16] have contributed to the service interruption gets repair immediately.

Many of real models could be considered by two phases of service is important, because some of the systems have more than one phase of service is considered. There is no work carried out in bulk service queue with extended Bernoulli vacation. So, we consider a batch arrival and bulk service is rendered by a minimum batch size of ' $a$ ' and a maximum of ' $b$ '. A single server providing two phases of service to a batch of customers with two dissimilar service rates. After attainment of two successive phases of service, if the server will go for the first type of vacation this assumed to be a probability $\theta$. In case, if the server has been continued to serve the next batch if exit with probability $1-\theta$. After attainment of the first type of vacation, if the server will go for the second type of extended vacation this assumed to be a probability $\mathrm{p}$. In case, if the server has been continued to serve the next batch if exit with probability 1-p. If the two phases of service get interrupted, it enters into the repair process of respective phases. At the end of the repair process, a batch of customer who was just being served before server breakdown waits for the remaining service to complete the service.

This paper is structured as follows. In section 2 the brief description of the mathematical model. In section 3, we present the definitions and equation governing of our system and also obtain the time dependent solution of our model. The steady state behavior of the system and the probability generating function of the queue size at a random epoch have been derived explicitly in section 4 and corresponding stability condition have been obtained in section 5. Also, we obtain the performance measures in the different states of the system, the mean queue size and the average waiting time in the queue explicitly in section 6. Section 7 deals with the queue size distribution at a departure epoch. Some particular cases are given in section 8. A numerical result and graphs are presented in section 9. Conclusions are given in section 10.

\section{Mathematical Description of The Queueing Model}

To describe the required queueing model, we assume the following.

- Customers arrive at the system in batches of variable size in a compound Poisson process and they are providing bulk service on a first come - first served basis. Let $\lambda c_{i} d t(i \geq 1)$ be the first order probability that a batch of $i$ customer arrives at the system during a short interval of time $(t, t+d t]$, where $0 \leq c_{i} \leq 1$ and $\sum_{i=1}^{\infty} c_{i}=1$ and $\lambda>0$ is the mean arrival rate of batches.

- There is a single server providing service to a batch of customers in two heterogeneous services in succession. The service time follows a general (arbitrary) distributions $B_{1}$ and $B_{2}$ at First Phase of Service (FPS) and Second Phase of Service (SPS) with rates of service $\mu_{1}$ and $\mu_{2}$, respectively. Let $B_{i}(v)$ and $b_{i}(v)$ be the distribution function and density function of the service time, respectively. The conditional probability density function of service completion during the interval $(x, x+d x]$, given that the elapsed service time is $x$, so that

$$
\mu_{i}(x)=\frac{b_{i}(x)}{1-B_{i}(x)}, i=1,2
$$

and, therefore

$$
b_{i}(v)=\mu_{i}(v) e^{-\int_{0}^{v} \mu_{i}(x) d x}, i=1,2
$$

- After attainment of Second Phase of Service (SPS), the server may take the first type of Bernoulli vacation with probability $\theta$, and with probability $(1-\theta)$ it waits for serving the next batch of 
customers. After completion of the first type of vacation, the server takes the second type of optional extended vacation with probability $\mathrm{p}$ or may continue to serve the next batch if exist, with probability $1-\mathrm{p}$.

- The vacation time of the server follows a general (arbitrary) distribution with distribution function $V_{i}(r)$ and density function $v_{i}(r)$. Let $\gamma_{i}(x) d x$ be the conditional probability of a completion of a vacation during the interval $(x, x+d x]$, given that the elapsed vacation time is $x$, so that

$$
\gamma_{i}(x)=\frac{v_{i}(x)}{1-V_{i}(x)}, i=1,2
$$

and, therefore

$$
v_{i}(r)=\gamma_{i}(r) e^{-\int_{0}^{r} \gamma_{i}(x) d x}, i=1,2
$$

- While the server is functioning with any phase of service, it may break down and is assumed to occur according to a Poisson stream with mean breakdown rates $\alpha_{1}$ for the First Phase of Service and $\alpha_{2}$ for the Second Phase of Service.

- If the service gets interrupted, the First phase of service (FPS) and a second phase of service (SPS) enter into the repair process of respective phases. Both phases of repair time follow general (arbitrary) distribution with distribution function $R_{i}(t)$ and the density function $r_{i}(t)$. Let $\beta_{i}(y) d y$ be the conditional probability of a completion of a repair time during the interval $(y, y+d y]$, given that the elapsed repair time is $y$, so that

$$
\beta_{i}(y)=\frac{d_{i}(y)}{1-R_{i}(y)}, i=1,2
$$

and therefore,

$$
r_{i}(t)=\beta_{i}(t) e^{-\int_{0}^{t} \beta_{i}(y) d y}, i=1,2
$$

- The customers are served according to the "First come-First served" discipline.

- Various stochastic processes involved in the system are assumed to be independent of each other.

\section{DEFINITIONS}

We define

1. $P_{i, n}(x, t)=$ Probability that at time $t$, the server is active providing $i^{\text {th }}$ phase of service $(i=1,2)$ and there are $n \quad(n \geq 0)$ customers in the queue excluding the batch being served and the elapsed service time on a batch of customers undergoing service is $x$. Accordingly, $P_{i, n}(t)=\int_{0}^{\infty} P_{i, n}(x, t) d x$ denotes the probability that at time $\mathrm{t}$ there are $\mathrm{n}$ customers in the queue excluding the batch of customers in $i^{\text {th }}$ phase of service without regard to the elapsed service time is $x$.

2. $V_{i, n}(x, t)=$ Probability that at a time $t$, the server is on $i^{\text {th }}$ type of vacation with elapsed vacation time is $x$ and there are $n(n \geq 0)$ customers in the queue. Accordingly, $V_{i, n}(t)=\int_{0}^{\infty} V_{i, n}(x, t) d x$ 
denotes the probability that at time t, there are $n$ customers in the queue and the server is on $i^{\text {th }}$ type of vacation without regard to the elapsed vacation time is $x$.

3. $R_{i, n}(x, y, t)=$ Probability that at time $t$, the server is under $i^{\text {th }}$ phase of repair $(i=1,2)$ (breakdown during $i^{\text {th }}$ phase of service time) with the elapsed service time on a batch of customers undergoing service is $x$, the elapsed repair time of server is $y$ and there are $n(n \geq 0)$ customers in the queue. Accordingly, $R_{i, n}(x, t)=\int_{0}^{\infty} R_{i, n}(x, y, t) d y$ denotes the probability that at time $t$ there are $n$ customers in the queue with the elapsed service time on a batch of customers undergoing service is $\mathrm{x}$ and without regard to the elapsed repair time is $y$.

4. $Q_{r}(t)=$ Probability that at time $t$, there are $r(0 \leq r \leq a-1)$ customers in the system and the server is idle but available in the system.

The Kolmogorov forward equations to govern the model under the transient state conditions for $i=1,2$; where sub index $i=1,2$ denotes the FPS and SPS respectively can be formulated as follows:

$\frac{\partial}{\partial x} P_{i, 0}(x, t)+\frac{\partial}{\partial t} P_{i, 0}(x, t)+\left(\lambda+\mu_{i}(x)+\alpha_{i}\right) P_{i, 0}(x, t)=\int_{0}^{\infty} R_{i, 0}(x, y, t) \beta_{i}(y) d y, i=1,2$

$$
\begin{aligned}
\frac{\partial}{\partial x} P_{i, n}(x, t)+\frac{\partial}{\partial t} P_{i, n}(x, t)+\left(\lambda+\mu_{i}(x)+\alpha_{i}\right) P_{i, n}(x, t)=\lambda \sum_{k=1}^{n} c_{k} P_{i, n-k}(x, t) & \\
& +\int_{0}^{\infty} R_{i, n}(x, y, t) \beta_{i}(y) d y, n \geq 1, i=1,2
\end{aligned}
$$

$\frac{\partial}{\partial x} V_{1,0}(x, t)+\frac{\partial}{\partial t} V_{1,0}(x, t)+\left(\lambda+\gamma_{1}(x)\right) V_{1,0}(x, t)=0$,

$$
\frac{\partial}{\partial x} V_{1, n}(x, t)+\frac{\partial}{\partial t} V_{1, n}(x, t)+\left(\lambda+\gamma_{1}(x)\right) V_{1, n}(x, t)=\lambda \sum_{k=1}^{n} c_{k} V_{1, n-k}(x, t), n \geq 1,
$$

$$
\frac{\partial}{\partial x} V_{2,0}(x, t)+\frac{\partial}{\partial t} V_{2,0}(x, t)+\left(\lambda+\gamma_{2}(x)\right) V_{2,0}(x, t)=0,
$$

$$
\frac{\partial}{\partial x} V_{2, n}(x, t)+\frac{\partial}{\partial t} V_{2, n}(x, t)+\left(\lambda+\gamma_{2}(x)\right) V_{2, n}(x, t)=\lambda \sum_{k=1}^{n} c_{k} V_{2, n-k}(x, t), n \geq 1,
$$

$$
\frac{\partial}{\partial y} R_{i, 0}(x, y, t)+\frac{\partial}{\partial t} R_{i, 0}(x, y, t)+\left(\lambda+\beta_{i}(y)\right) R_{i, 0}(x, y, t)=0, i=1,2
$$

$$
\frac{\partial}{\partial y} R_{i, n}(x, y, t)+\frac{\partial}{\partial t} R_{i, n}(x, y, t)+\left(\lambda+\beta_{i}(y)\right) R_{i, n}(x, y, t)=\lambda \sum_{k=1}^{n} c_{k} R_{i, n-k}(x, y, t), n \geq 1, i=1,2
$$

$$
\frac{d}{d t} Q_{0}(t)=-\lambda Q_{0}(t)+(1-\theta) \int_{0}^{\infty} P_{2,0}(x, t) \mu_{2}(x) d x+(1-p) \int_{0}^{\infty} V_{1,0}(x, t) \gamma_{1}(x) d x+\int_{0}^{\infty} V_{2,0}(x, t) \gamma_{2}(x) d x,
$$

$$
\begin{array}{r}
\frac{d}{d t} Q_{r}(t)=-\lambda Q_{r}(t)+\lambda \sum_{k=1}^{r} c_{k} Q_{r-k}(t)+(1-\theta) \int_{0}^{\infty} P_{2, r}(x, t) \mu_{2}(x) d x+(1-p) \int_{0}^{\infty} V_{1, r}(x, t) \gamma_{1}(x) d x \\
+\int_{0}^{\infty} V_{2, r}(x, t) \gamma_{2}(x) d x, 1 \leq r \leq a-1 .
\end{array}
$$


To solve the equations (1) to (10), the following boundary conditions at $x=0$ and $y=0$ are considered,

$$
\begin{aligned}
& P_{1,0}(0, t)=\lambda \sum_{r=a}^{b} \sum_{k=0}^{a-1} c_{r-k} Q_{k}(t)+(1-\theta) \sum_{r=a}^{b} \int_{0}^{\infty} P_{2, r}(x, t) \mu_{2}(x) d x+(1-p) \sum_{r=a}^{b} \int_{0}^{\infty} V_{1, r}(x, t) \gamma_{1}(x) d x \\
& +\sum_{r=a}^{b} \int_{0}^{\infty} V_{2, r}(x, t) \gamma_{2}(x) d x \\
& P_{1, n}(0, t)=\lambda \sum_{k=0}^{a-1} c_{b+n-k} Q_{k}(t)+(1-\theta) \int_{0}^{\infty} P_{2, n+b}(x, t) \mu_{2}(x) d x+(1-p) \int_{0}^{\infty} V_{1, n+b}(x, t) \gamma_{1}(x) d x \\
& +\int_{0}^{\infty} V_{2, n+b}(x, t) \gamma_{2}(x) d x, n \geq 1 \\
& P_{2, n}(0, t)=\int_{0}^{\infty} P_{1, n}(x, t) \mu_{1}(x) d x, n \geq 0 \\
& V_{1, n}(0, t)=\theta \int_{0}^{\infty} P_{2, n}(x, t) \mu_{2}(x) d x, n \geq 0 \\
& V_{2, n}(0, t)=p \int_{0}^{\infty} V_{1, n}(x, t) \gamma_{1}(x) d x, n \geq 0 \\
& R_{i, n}(x, 0, t)=\alpha_{i} P_{i, n}(x, t), n \geq 0, i=1,2 .
\end{aligned}
$$

Further, it is assume that initially there are no adequate number of customers in the system and the server is idle. So the initial conditions are

$$
\begin{aligned}
& Q_{0}(0)=1, Q_{r}(0)=0 \text { for } 1 \leq r \leq a-1, \\
& P_{i, n}(0)=R_{i, n}(0)=V_{1, n}(0)=V_{2, n}(0)=0 \text { for } n \geq 0, i=1,2 .
\end{aligned}
$$

To solve the above equations, let us introduce the following probability generating functions for $i=1,2$ and $|z| \leq 1$

$$
\begin{aligned}
& P_{i}(x, z, t)=\sum_{n=0}^{\infty} z^{n} P_{i, n}(x, t) ; P_{i}(z, t)=\sum_{n=0}^{\infty} z^{n} P_{i, n}(t) \\
& V_{1}(x, z, t)=\sum_{n=0}^{\infty} z^{n} V_{1, n}(x, t) ; V_{1}(z, t)=\sum_{n=0}^{\infty} z^{n} V_{1, n}(t) \\
& V_{2}(x, z, t)=\sum_{n=0}^{\infty} z^{n} V_{2, n}(x, t) ; V_{2}(z, t)=\sum_{n=0}^{\infty} z^{n} V_{2, n}(t) \\
& R_{i}(x, y, z, t)=\sum_{n=0}^{\infty} z^{n} R_{i, n}(x, y, t) ; R_{i}(x, z, t)=\sum_{n=0}^{\infty} z^{n} R_{i, n}(x, t) \\
& C(z)=\sum_{n=1}^{\infty} c_{n} z^{n} ; Q(z)=\sum_{r=0}^{a-1} Q_{r} z^{r} .
\end{aligned}
$$

Define the Laplace transform of a function $f(t)$ as 


$$
\bar{f}(s)=\int_{0}^{\infty} e^{-s t} f(t) d t, \mathfrak{R}(s)>0
$$

Taking the Laplace transform of equations (1) to (16) and using (17), we get

$$
\begin{aligned}
& \frac{\partial}{\partial x} \bar{P}_{i, 0}(x, s)+\left(s+\lambda+\mu_{i}(x)+\alpha_{i}\right) \bar{P}_{i, 0}(x, s)=\int_{0}^{\infty} \bar{R}_{i, 0}(x, y, s) \beta_{i}(y) d y, i=1,2 \\
& \frac{\partial}{\partial x} \bar{P}_{i, n}(x, s)+\left(s+\lambda+\mu_{i}(x)+\alpha_{i}\right) \bar{P}_{i, n}(x, s)=\lambda \sum_{k=1}^{n} c_{k} \bar{P}_{i, n-k}(x, s) \\
&+\int_{0}^{\infty} \bar{R}_{i, n}(x, y, s) \beta_{i}(y) d y, n \geq 1, i=1,2
\end{aligned}
$$

$\frac{\partial}{\partial x} \bar{V}_{1,0}(x, s)+\left(s+\lambda+\gamma_{1}(x)\right) \bar{V}_{1,0}(x, s)=0$,

$\frac{\partial}{\partial x} \bar{V}_{1, n}(x, s)+\left(s+\lambda+\gamma_{1}(x)\right) \bar{V}_{1, n}(x, s)=\lambda \sum_{k=1}^{n} c_{k} \bar{V}_{1, n-k}(x, s), n \geq 1$,

$\frac{\partial}{\partial x} \bar{V}_{2,0}(x, s)+\left(s+\lambda+\gamma_{2}(x)\right) \bar{V}_{2,0}(x, s)=0$,

$$
\frac{\partial}{\partial x} \bar{V}_{2, n}(x, s)+\left(s+\lambda+\gamma_{2}(x)\right) \bar{V}_{2, n}(x, s)=\lambda \sum_{k=1}^{n} c_{k} \bar{V}_{2, n-k}(x, s), n \geq 1,
$$

$$
\frac{\partial}{\partial y} \bar{R}_{i, 0}(x, y, s)+\left(s+\lambda+\beta_{i}(y)\right) \bar{R}_{i, 0}(x, y, s)=0, i=1,2
$$

$\frac{\partial}{\partial y} \bar{R}_{i, n}(x, y, s)+\left(s+\lambda+\beta_{i}(y)\right) \bar{R}_{i, n}(x, y, s)=\lambda \sum_{k=1}^{n} c_{k} \bar{R}_{i, n-k}(x, y, s), \quad n \geq 1, i=1,2$

$$
(s+\lambda) \bar{Q}_{0}(s)=1+(1-\theta) \int_{0}^{\infty} \bar{P}_{2,0}(x, s) \mu_{2}(x) d x
$$

$$
+(1-p) \int_{0}^{\infty} \bar{V}_{1,0}(x, s) \gamma_{1}(x) d x+\int_{0}^{\infty} \bar{V}_{2,0}(x, s) \gamma_{2}(x) d x
$$

$$
\begin{aligned}
(s+\lambda) \bar{Q}_{r}(s)=\lambda \sum_{k=1}^{r} c_{k} \bar{Q}_{r-k}(s) & +(1-\theta) \int_{0}^{\infty} \bar{P}_{2, r}(x, s) \mu_{2}(x) d x \\
& +(1-p) \int_{0}^{\infty} \bar{V}_{1, r}(x, s) \gamma_{1}(x) d x+\int_{0}^{\infty} \bar{V}_{2, r}(x, s) \gamma_{2}(x) d x, \quad 1 \leq r \leq a-1 .
\end{aligned}
$$

$$
\begin{array}{r}
\bar{P}_{1,0}(0, s)=\lambda \sum_{r=a}^{b} \sum_{k=0}^{a-1} c_{r-k} \bar{Q}_{k}(s)+(1-\theta) \sum_{r=a}^{b} \int_{0}^{\infty} \bar{P}_{2, r}(x, s) \mu_{2}(x) d x+(1-p) \sum_{r=a}^{b} \int_{0}^{\infty} \bar{V}_{1, r}(x, s) \gamma_{1}(x) d x \\
+\sum_{r=a}^{b} \int_{0}^{\infty} \bar{V}_{2, r}(x, s) \gamma_{2}(x) d x,
\end{array}
$$




$$
\begin{aligned}
& \bar{P}_{1, n}(0, s)=\lambda \sum_{k=0}^{a-1} c_{b+n-k} \bar{Q}_{k}(s)+(1-\theta) \int_{0}^{\infty} \bar{P}_{2, n+b}(x, s) \mu_{2}(x) d x+(1-p) \int_{0}^{\infty} \bar{V}_{1, n+b}(x, s) \gamma_{1}(x) d x \\
& \quad+\int_{0}^{\infty} \bar{V}_{2, n+b}(x, s) \gamma_{2}(x) d x, n \geq 1 \\
& \bar{P}_{2, n}(0, s)=\int_{0}^{\infty} \bar{P}_{1, n}(x, s) \mu_{1}(x) d x, n \geq 0 \\
& \bar{V}_{1, n}(0, s)=\theta \int_{0}^{\infty} \bar{P}_{2, n}(x, s) \mu_{2}(x) d x, n \geq 0 \\
& \bar{V}_{2, n}(0, s)=p \int_{0}^{\infty} \bar{V}_{1, n}(x, s) \gamma_{1}(x) d x, n \geq 0 \\
& \bar{R}_{i, n}(x, 0, s)=\alpha_{i} \bar{P}_{i, n}(x, s), n \geq 0, i=1,2 .
\end{aligned}
$$

By multiplying equations (21),(23),(25) and (27) by $z^{n}$ and then taking summation over all possible values of $n$, adding to the equations (20), (22),(24) and (26) respectively, and using the generating functions defined in (18), we get

$$
\begin{aligned}
& \frac{\partial}{\partial x} \bar{P}_{i}(x, z, s)+\left(s+\lambda(1-C(z))+\mu_{i}(x)+\alpha_{i}\right) \bar{P}_{i}(x, z, s)=\int_{0}^{\infty} \bar{R}_{i}(x, y, z, s) \beta_{i}(y) d y, i=1,2 \\
& \frac{\partial}{\partial x} \bar{V}_{1}(x, z, s)+\left(s+\lambda(1-C(z))+\gamma_{1}(x)\right) \bar{V}_{1}(x, z, s)=0, \\
& \frac{\partial}{\partial x} \bar{V}_{2}(x, z, s)+\left(s+\lambda(1-C(z))+\gamma_{2}(x)\right) \bar{V}_{2}(x, z, s)=0, \\
& \frac{\partial}{\partial y} \bar{R}_{i}(x, y, z, s)+\left(s+\lambda(1-C(z))+\beta_{i}(y)\right) \bar{R}_{i}(x, y, z, s)=0, i=1,2 .
\end{aligned}
$$

Multiplying both sides of equation (31) by $z^{n}$ summing over $n$ from 0 to $\infty$, and use the equation (30), we get

$$
\begin{aligned}
z^{b} \bar{P}_{1}(0, z, s)= & \lambda \sum_{r=0}^{a-1} \sum_{n=1}^{b-r-1} c_{n} \bar{Q}_{r}(s)\left(z^{b}-z^{n+r}\right)-z^{b} \sum_{r=0}^{a-1}(s+\lambda) \bar{Q}_{r}(s)+\lambda \sum_{r=0}^{a-1} C(z) \bar{Q}_{r}(s) z^{r}+z^{b} \\
+ & (1-\theta) \int_{0}^{\infty} \bar{P}_{2}(x, z, s) \mu_{2}(x) d x+(1-p) \int_{0}^{\infty} \bar{V}_{1}(x, z, s) \gamma_{1}(x) d x \\
& +\int_{0}^{\infty} \bar{V}_{2}(x, z, s) \gamma_{2}(x) d x+(1-\theta) \sum_{r=0}^{b-1}\left(z^{b}-z^{r}\right) \int_{0}^{\infty} \bar{P}_{2, r}(x, s) \mu_{2}(x) d x \\
+ & (1-p) \sum_{r=0}^{b-1}\left(z^{b}-z^{r}\right) \int_{0}^{\infty} \bar{V}_{1, r}(x, s) \gamma_{1}(x) d x+\sum_{r=0}^{b-1}\left(z^{b}-z^{r}\right) \int_{0}^{\infty} \bar{V}_{2, r}(x, s) \gamma_{2}(x) d x,
\end{aligned}
$$

Similarly from equations (32),(33) (34) and (35), we get

$$
\begin{aligned}
& \bar{P}_{2}(0, z, s)=\bar{P}_{1}(0, z, s) \bar{B}_{1}\left(\psi_{1}(z, s)\right), \\
& \bar{V}_{1}(0, z, s)=\theta \bar{P}_{1}(0, z, s) \bar{B}_{1}\left(\psi_{1}(z, s)\right) \bar{B}_{2}\left(\psi_{2}(z, s)\right),
\end{aligned}
$$


Analysis of $M^{X} / \mathrm{G}(\mathbf{a}, \mathrm{b}) / 1$ Queueing System with Two Phases of Service Subject to Server Breakdown and Extended Bernoulli Vacations

$\bar{V}_{2}(0, z, s)=\theta p \bar{P}_{1}(0, z, s) \bar{B}_{1}\left(\psi_{1}(z, s)\right) \bar{B}_{2}\left(\psi_{2}(z, s)\right) \bar{V}_{1}(\phi(z, s))$,

$\bar{R}_{i}(x, 0, z, s)=\alpha_{i} \bar{P}_{i}(x, z, s), i=1,2$.

Solving the partial differential equations (36) to (39), it follows that

$$
\begin{aligned}
& \bar{P}_{i}(x, z, s)=\bar{P}_{i}(0, z, s) e^{-\psi_{i}(z, s) x-\int_{0}^{x} \mu_{i}(t) d t}, i=1,2 \\
& \bar{V}_{1}(x, z, s)=\bar{V}_{1}(0, z, s) e^{-\phi(z, s) x-\int_{0}^{x} \gamma_{1}(t) d t}, \\
& \bar{V}_{2}(x, z, s)=\bar{V}_{2}(0, z, s) e^{-\phi(z, s) x-\int_{0}^{x} \gamma_{2}(t) d t}, \\
& \bar{R}_{i}(x, y, z, s)=\bar{R}_{i}(x, 0, z, s) e^{-\phi(z, s) y-\int_{0}^{y} \beta_{i}(t) d t}, i=1,2 .
\end{aligned}
$$

Integrating equation (48) from 0 to $\infty$ with respect to $y$, we get for $i=1,2$

$$
\begin{aligned}
& \bar{R}_{i}(x, z, s)=\int_{0}^{\infty} \bar{R}_{i}(x, y, z, s) d y \\
& \bar{R}_{i}(x, z, s)=\bar{R}_{i}(x, 0, z, s)\left[\frac{1-\bar{R}_{i}(\phi(z, s))}{\phi(z, s)}\right]
\end{aligned}
$$

Now multiplying both sides of equations (45) to (48) by $\mu_{i}(x), \gamma_{1}(x), \gamma_{2}(x)$, and $\beta_{i}(y)$ respectively, and integrating, we obtain

$$
\begin{aligned}
& \int_{0}^{\infty} \bar{P}_{i}(x, z, s) \mu_{i}(x) d x=\bar{P}_{i}(0, z, s) \bar{B}_{i}\left(\psi_{i}(z, s)\right) \\
& \int_{0}^{\infty} \bar{V}_{1}(x, z, s) \gamma_{1}(x) d x=\bar{V}_{1}(0, z, s) \bar{V}_{1}(\phi(z, s)) \\
& \int_{0}^{\infty} \bar{V}_{2}(x, z, s) \gamma_{2}(x) d x=\bar{V}_{2}(0, z, s) \bar{V}_{2}(\phi(z, s)) \\
& \int_{0}^{\infty} \bar{R}_{i}(x, y, z, s) \beta_{i}(y) d y=\bar{R}_{i}(x, 0, z, s) \bar{R}_{i}(\phi(z, s))
\end{aligned}
$$

where

$$
\bar{B}_{i}\left(\psi_{i}(z, s)\right)=\int_{0}^{\infty} e^{-\psi_{i}(z, s) x} d B_{i}(x)
$$




$$
\begin{aligned}
& \bar{V}_{1}(\phi(z, s))=\int_{0}^{\infty} e^{-\phi(z, s) x} d V_{1}(x) \\
& \bar{V}_{2}(\phi(z, s))=\int_{0}^{\infty} e^{-\phi(z, s) x} d V_{2}(x) \\
& \bar{R}_{i}(\phi(z, s))=\int_{0}^{\infty} e^{-\phi(z, s) y} d R_{i}(y)
\end{aligned}
$$

are the Laplace-Stieltjes transform of the service time $B_{i}(x)$, vacation times $V_{1}(x)$ and $V_{2}(x)$ and repair time $R_{i}(y)$, respectively.

Again integrating equations (45), (46),(47) and (49) by parts with respect to $\mathrm{x}$ and using the equation (41),(42),(43), and (44), we get for $\mathrm{i}=1,2$

$$
\begin{aligned}
& \bar{P}_{1}(z, s)=\bar{P}_{1}(0, z, s)\left[\frac{\left.1-\bar{B}_{1}\left(\psi_{1}(z, s)\right)\right]}{\psi_{1}(z, s)}\right] \\
& \bar{P}_{2}(z, s)=\bar{P}_{1}(0, z, s) \bar{B}_{1}\left(\psi_{1}(z, s)\right)\left[\frac{1-\bar{B}_{2}\left(\psi_{2}(z, s)\right)}{\psi_{2}(z, s)}\right], \\
& \bar{V}_{1}(z, s)=\theta \bar{P}_{1}(0, z, s) \bar{B}_{1}\left(\psi_{1}(z, s)\right) \bar{B}_{2}\left(\psi_{2}(z, s)\right)\left[\frac{1-\bar{V}_{1}(\phi(z, s))}{\phi(z, s)}\right], \\
& \bar{V}_{2}(z, s)=\theta p \bar{P}_{1}(0, z, s) \bar{B}_{1}\left(\psi_{1}(z, s)\right) \bar{B}_{2}\left(\psi_{2}(z, s)\right) \bar{V}_{1}(\phi(z, s))\left[\frac{1-\bar{V}_{2}(\phi(z, s))}{\phi(z, s)}\right], \\
& \bar{R}_{1}(z, s)=\alpha_{1} \bar{P}_{1}(0, z, s)\left[\frac{1-\bar{B}_{1}\left(\psi \psi_{1}(z, s)\right)}{\psi_{1}(z, s)}\left[\frac{1-\bar{R}_{1}(\phi(z, s))}{\phi(z, s)}\right],\right. \\
& \bar{R}_{2}(z, s)=\alpha_{2} \bar{P}_{1}(0, z, s) \bar{B}_{1}\left(\psi_{1}(z, s)\right)\left[\frac{1-\bar{B}_{2}\left(\psi_{2}(z, s)\right)}{\psi_{2}(z, s)}\right]\left[\frac{1-\bar{R}_{2}(\phi(z, s))}{\phi(z, s)}\right] .
\end{aligned}
$$

Inserting the equations (50),(51) and (52) into the equation (40), we get

$$
\begin{aligned}
& \bar{P}_{1}(0, z, s)=\left[\begin{array}{c}
\lambda \sum_{r=0}^{a-1} C(z) \bar{Q}_{r}(s) z^{r}-z^{b}(s+\lambda) \sum_{r=0}^{a-1} \bar{Q}_{r}(s) \\
+z^{b}+\lambda \sum_{r=0}^{a-1} \sum_{n=1}^{b-r-1} c_{n} \bar{Q}_{r}(s)\left(z^{b}-z^{n+r}\right) \\
+\sum_{r=0}^{b-1}\left(z^{b}-z^{r}\right)\left(\int_{0}^{\infty} \bar{V}_{2, r}(x, s) \gamma_{2}(x) d x\right. \\
+(1-p) \int_{0}^{\infty} \bar{V}_{1, r}(x, s) \gamma_{1}(x) d x \\
\left.+(1-\theta) \int_{0}^{\infty} \bar{P}_{2, r}(x, s) \mu_{2}(x) d x\right)
\end{array}\right] . \\
& \text { where } \quad \phi(z, s)=s+\lambda(1-C(z)), \quad \psi_{i}(z, s)=\phi(z, s)+\alpha_{i}\left(1-\bar{R}_{i}(\phi(z, s))\right), i=1,2 \\
& K(z, s)=(1-p) \bar{V}_{1}(\phi(z, s))+p \bar{V}_{1}(\phi(z, s)) \bar{V}_{2}(\phi(z, s))
\end{aligned}
$$


Analysis of $M^{X} / \mathrm{G}(\mathrm{a}, \mathrm{b}) / 1$ Queueing System with Two Phases of Service Subject to Server Breakdown and Extended Bernoulli Vacations

Substituting the equation (60) into the equations (54),(55),(56),(57),(58) and (59) and taking the inverse laplace transform of these equations, we get the probability generating fuctions of various states of the system are determined under transient state.

\section{The STEADY STATE Results}

In this section, we shall derive the steady state probability distribution for our queueing model. To define the steady state probabilities, we suppress the argument $t$ wherever it appears in the timedependent analysis. This can be obtained by applying the Tauberian property,

$$
\lim _{s \rightarrow 0} s \bar{f}(s)=\lim _{t \rightarrow \infty} f(t) .
$$

The PGF of the server's state queue size distribution under the steady state conditions are given by

$$
\begin{aligned}
& P_{1}(z)=P_{1}(0, z)\left[\frac{1-\bar{B}_{1}\left(\psi_{1}(z)\right)}{\psi_{1}(z)}\right], \\
& P_{2}(z)=P_{1}(0, z) \bar{B}_{1}\left(\psi_{1}(z)\left[\frac{1-\bar{B}_{2}\left(\psi_{2}(z)\right)}{\psi_{2}(z)}\right],\right. \\
& V_{1}(z)=\theta P_{1}(0, z) \bar{B}_{1}\left(\psi_{1}(z)\right) \bar{B}_{2}\left(\psi_{2}(z)\right)\left[\frac{1-\bar{V}_{1}(\phi(z))}{\phi(z)}\right], \\
& V_{2}(z)=\theta p P_{1}(0, z) \bar{B}_{1}\left(\psi_{1}(z)\right) \bar{B}_{2}\left(\psi_{2}(z)\right) \bar{V}_{1}(\phi(z))\left[\frac{1-\bar{V}_{2}(\phi(z))}{\phi(z)}\right], \\
& R_{1}(z)=\alpha_{1} P_{1}(0, z)\left[\frac{1-\bar{B}_{1}\left(\psi_{1}(z)\right)}{\psi_{1}(z)}\right]\left[\frac{1-\bar{R}_{1}(\phi(z))}{\phi(z)}\right], \\
& R_{2}(z)=\alpha_{2} P_{1}(0, z) \bar{B}_{1}\left(\psi_{1}(z)\right)\left[\frac{1-\bar{B}_{2}\left(\psi_{2}(z)\right)}{\psi_{2}(z)}\right]\left[\frac{1-\bar{R}_{2}(\phi(z))}{\phi(z)}\right],
\end{aligned}
$$

where

$$
P_{1}(0, z)=\frac{\left(\begin{array}{c}
\lambda \sum_{r=0}^{a-1} Q_{r}\left(C(z) z^{r}-z^{b}\right)+\lambda \sum_{r=0}^{a-1} \sum_{n=1}^{b-r-1} c_{n} Q_{r}\left(z^{b}-z^{n+r}\right) \\
+\sum_{r=0}^{b-1}\left(z^{b}-z^{r}\right)\left(\int_{0}^{\infty} \bar{V}_{2, r}(x) \gamma_{2}(x) d x\right. \\
+(1-p) \int_{0}^{\infty} \bar{V}_{1, r}(x) \gamma_{1}(x) d x \\
\left.+(1-\theta) \int_{0}^{\infty} \bar{P}_{2, r}(x) \mu_{2}(x) d x\right)
\end{array}\right]}{\left[z^{b}-((1-\theta)+\theta K(z)) \bar{B}_{1}\left(\psi_{1}(z)\right) \bar{B}_{2}\left(\psi_{2}(z)\right)\right]} .
$$

\subsection{Queue Size Distribution at a Random Epoch}

By adding (62),(63),(64),(65),(66) and (67) with idle term, we get the PGF of the queue size distribution at a random epoch.

$$
P(z)=P_{1}(z)+P_{2}(z)+V_{1}(z)+V_{2}(z)+R_{1}(z)+R_{2}(z)+Q(z)
$$




$$
P(z)=\frac{\left(\begin{array}{l}
{\left[\lambda \sum_{r=0}^{a-1} Q_{r}\left(C(z) z^{r}-z^{b}\right)+\lambda \sum_{r=0}^{a-1} \sum_{n=1}^{b-r-1} c_{n} Q_{r}\left(z^{b}-z^{n+r}\right)+\sum_{r=0}^{b-1}\left(z^{b}-z^{r}\right) W_{r}\right]} \\
\times\left[\phi(z)\left(1-\bar{B}_{1}\left(\psi_{1}(z)\right)\right) \psi_{2}(z)+\phi(z) \psi_{1}(z) \bar{B}_{1}\left(\psi_{1}(z)\right)\left(1-\bar{B}_{2}\left(\psi_{2}(z)\right)\right)\right. \\
+\theta \psi_{1}(z) \psi_{2}(z) \bar{B}_{1}\left(\psi_{1}(z)\right) \bar{B}_{2}\left(\psi_{2}(z)\right)\left(1-\bar{V}_{1}(\phi(z))\right) \\
+\theta p \psi_{1}(z) \psi_{2}(z) \bar{B}_{1}\left(\psi_{1}(z)\right) \bar{B}_{2}\left(\psi_{1}(z)\right) \bar{V}_{1}(\phi(z)) \times\left(1-\bar{V}_{2}(\phi(z))\right) \\
+\alpha_{1} \psi_{2}(z)\left(1-\bar{B}_{1}\left(\psi_{1}(z)\right)\right)\left(1-\bar{R}_{1}(\phi(z))\right) \\
\left.+\alpha_{2} \psi_{1}(z) \bar{B}_{1}\left(\psi_{1}(z)\right)\left(1-\bar{B}_{2}\left(\psi_{2}(z)\right)\right)\left(1-\bar{R}_{2}(\phi(z))\right)\right] \\
+\left[z^{b}-((1-\theta)+\theta K(z)) \bar{B}_{1}\left(\psi_{1}(z)\right) \bar{B}_{2}\left(\psi_{2}(z)\right)\right] \times\left[\phi(z) \psi_{1}(z) \psi_{2}(z) Q(z)\right]
\end{array}\right)}{\left[z^{b}-((1-\theta)+\theta K(z)) \bar{B}_{1}\left(\psi_{1}(z)\right) \bar{B}_{2}\left(\psi_{2}(z)\right)\right] \times\left[\phi(z) \psi_{1}(z) \psi_{2}(z)\right]}
$$

where

$$
\begin{array}{lc}
\phi(z)=\lambda(1-C(z)) ; & \psi_{1}(z)=\phi(z)+\alpha_{1}\left(1-\bar{R}_{1}(\phi(z))\right) \\
\psi_{2}(z)=\phi(z)+\alpha_{2}\left(1-\bar{R}_{2}(\phi(z))\right) ; & K(z)=(1-p) \bar{V}_{1}(\phi(z))+p \bar{V}_{1}(\phi(z)) \bar{V}_{2}(\phi(z)) \\
W_{r}=(1-\theta) \int_{0}^{\infty} P_{2, r}(x) \mu_{2}(x) d x+V_{r} ; & V_{r}=(1-p) \int_{0}^{\infty} V_{1, r}(x) \gamma_{1}(x) d x+\int_{0}^{\infty} V_{2, r}(x) \gamma_{2}(x) d x
\end{array}
$$

\section{Stability Condition}

The probability generating function has to satisfy $\mathrm{P}(1)=1$. In order to satisfy this condition, apply L'Hopital rules and equating the expression to 1 , we get

$$
\begin{aligned}
& X_{1} \times\left[A_{1}+\theta E\left(V_{1}\right)+\theta p E\left(V_{2}\right)\right]+\left[b-\lambda E(I)\left(A_{1}+\theta E\left(V_{1}\right)+\theta p E\left(V_{2}\right)\right)\right] \times \sum_{r=0}^{a-1} Q_{r} \\
& =\left[b-\lambda E(I)\left(A_{1}+\theta E\left(V_{1}\right)+\theta p E\left(V_{2}\right)\right)\right]
\end{aligned}
$$

Next, we calculate the unknown probabilities, $W_{r}, r=0,1,2, \ldots b-1$ and then these are related to the idle-server probabilities, $Q_{r}, \quad r=0,1,2, \ldots a-1$, then the left hand side of the above expression must be positive. Thus $\mathrm{P}(1)=1$ is satisfied if

$$
\begin{aligned}
& \left(z^{b}-((1-\theta)+\theta K(z)) \bar{B}_{1}\left(\psi_{1}(z)\right) \bar{B}_{2}\left(\psi_{2}(z)\right)\right)>0 . \\
& \text { If } \rho=\frac{\left[\lambda E(I)\left(A_{1}+\theta E\left(V_{1}\right)+\theta p E\left(V_{2}\right)\right)\right]}{b} \text { then } \rho<1
\end{aligned}
$$

is the condition to be satisfied for the existence of steady state for the model under consideration. Equation (69) has b+a unknowns. Using the following result, we can express $W_{r}$ in terms of $Q_{r}$ in such a way that numerator have only ' $b$ ' constants. Now equation (69) gives the PGF of the number of customers involving ' $\mathrm{b}$ ' unknowns. By Rouche's theorem, the expression $\left[z^{b}-((1-\theta)+\theta K(z)) \bar{B}_{1}\left(\psi_{1}(z)\right) \bar{B}_{2}\left(\psi_{2}(z)\right)\right]$ has $b-1$ zeros inside and one on the unit circle $|z|=1$ . Since $\mathrm{P}(\mathrm{z})$ is analytic within and on the unit circle, the numerator must vanish at these points, which 
Analysis of $M^{X} / \mathrm{G}(\mathrm{a}, \mathrm{b}) / 1$ Queueing System with Two Phases of Service Subject to Server Breakdown and Extended Bernoulli Vacations

gives ' $b$ ' equations in ' $b$ ' unknowns. These equations can be solved by any suitable numerical technique.

5.1 Result: Let $W_{r}$ can be expressed in terms of $Q_{r}$ as

$$
\sum_{r=0}^{a-1} W_{r}=\lambda \sum_{r=0}^{a-1} Q_{r}-\lambda \sum_{r=0}^{a-1} Q_{r} \sum_{k=1}^{a-r-1} c_{k}
$$

Where, $W_{r}$ is the probabilities of the ' $\mathrm{r}$ ' customers in the queue during idle period and $X_{1}, A_{1}$, $E(I)$ are given in Section 6.

\section{Performance Measures}

In this section, we derive some system probabilities, the mean number of customers in the queue $\left(L_{q}\right)$ and the average time a customer spends in the queue $\left(W_{q}\right)$. From (71) we have $\rho<1$, which is stability condition.

\subsection{System state probabilities}

From equation (62) to (67), by setting $z \rightarrow 1$ and applying L'Hopital's rule whenever necessary, we get the following results

- Let $P_{q}(1)$ be the steady state probability that the server is busy

$$
P_{q}(1)=P_{1}(1)+P_{2}(1)=\frac{\left(X_{1} \times\left[E\left(B_{1}\right)+E\left(B_{2}\right)\right]\right)}{\left[b-\lambda E(I)\left(A_{1}+\theta E\left(V_{1}\right)+\theta p E\left(V_{2}\right)\right)\right]}
$$

- Let $V_{q}(1)$ be the steady state probability that the server is on vacation

$$
V_{q}(1)=V_{1}(1)+V_{2}(1)=\frac{\left(X_{1} \times\left[\theta E\left(v_{1}\right)+\theta p E\left(V_{2}\right)\right]\right)}{\left[b-\lambda E(I)\left(A_{1}+\theta E\left(V_{1}\right)+\theta p E\left(V_{2}\right)\right)\right]}
$$

- Let $R_{q}(1)$ be the steady state probability that the server is under repair

$$
R_{q}(1)=R_{1}(1)+R_{2}(1)=\frac{\left(X_{1} \times\left[\alpha_{1} E\left(R_{1}\right) E\left(B_{1}\right)+\alpha_{2} E\left(R_{2}\right) E\left(B_{2}\right)\right]\right)}{\left[b-\lambda E(I)\left(A_{1}+\theta E\left(V_{1}\right)+\theta p E\left(V_{2}\right)\right)\right]}
$$

\subsection{Mean Queue Size}

- The mean number of customers in the queue $\left(L_{q}\right)$ under steady state condition is obtained by differentiating (69) with respect to $\mathrm{z}$ and evaluating at $z=1$.

$$
\begin{aligned}
& L_{q}=\lim _{z \rightarrow 1} \frac{d}{d z} P(z) \\
& P^{\prime}(1)=\left[\frac{N^{V}(1) D^{I V}(1)-D^{V}(1) N^{I V}(1)}{5\left[\left(D^{I V}\right)^{2}\right]}\right]
\end{aligned}
$$

where 


$$
\begin{aligned}
& D^{I V}=-24(\lambda E(I))^{3} T_{1} T_{2}\left(b-\lambda E(I)\left(A_{1}+\theta E\left(V_{1}\right)+\theta p E\left(V_{2}\right)\right)\right) \\
& D^{V}=-60(\lambda E(I))^{2}\left[\left(b(b-1)-2 \theta p(\lambda E(I))^{2} E\left(V_{1}\right) E\left(V_{2}\right)-\theta p \lambda E(I(I-1))\left(E\left(V_{1}\right)+E\left(V_{2}\right)\right.\right.\right. \\
& -\theta p(\lambda E(I))^{2}\left(E\left(V_{1}^{2}\right)+E\left(V_{2}^{2}\right)\right)-\theta(1-p) C_{1} \\
& -2 \theta(\lambda E(I))^{2}\left(E\left(V_{1}\right)+p E\left(V_{2}\right)\right)\left(T_{1} E\left(B_{1}\right)+T_{2} E\left(B_{2}\right)\right) \\
& -2(\lambda E(I))^{2} T_{1} T_{2} E\left(B_{1}\right) E\left(B_{2}\right)-S_{1} E\left(B_{1}\right)-S_{2} E\left(B_{2}\right) \\
& \left.-(\lambda E(I))^{2}\left[T_{1}^{2} E\left(B_{1}^{2}\right)+T_{2}^{2} E\left(B_{2}^{2}\right)\right]\right) \times\left(\lambda E(I) T_{1} T_{2}\right) \\
& +\left(b-\lambda E(I)\left(A_{1}+\theta E\left(V_{1}\right)+\theta p E\left(V_{2}\right)\right)\right) \times\left[3 T_{1} T_{2} E(I(I-1))+T_{2} \alpha_{1}(\lambda E(I))^{2} E\left(R_{1}^{2}\right)\right. \\
& \left.\left.+T_{1} \alpha_{2}(\lambda E(I))^{2} E\left(R_{2}^{2}\right)\right]\right] \\
& N^{I V}=-24(\lambda E(I))^{3} T_{1} T_{2}\left[X_{1}\left(A_{1}+\theta E\left(v_{1}\right)+\theta p E\left(v_{2}\right)\right)+\left(b-\lambda E(I)\left(A_{1}+\theta E\left(v_{1}\right)+\theta p E\left(v_{2}\right)\right)\right) \sum_{r=0}^{a-1} Q_{r}\right] \\
& N^{V}=-60(\lambda E(I))^{2}\left[X_{2} \lambda E(I) T_{1} T_{2}\left(A_{1}+\theta E\left(v_{1}\right)+\theta p E\left(v_{2}\right)\right)\right. \\
& +X_{1}\left(T_{2} E\left(B_{1}\right) S_{1}+T_{2}\left(\lambda E(I) T_{1}\right)^{2} E\left(B_{1}^{2}\right)\right. \\
& +\lambda E(I(I-1)) T_{1} T_{2} E\left(B_{1}\right)+2(\lambda E(I))^{2} T_{1}^{2} T_{2} E\left(B_{1}\right) E\left(B_{2}\right) \\
& +T_{1} S_{2}\left(E\left(B_{1}\right)+E\left(B_{2}\right)\right)+T_{2} E\left(B_{2}\right)\left[T_{1} \lambda E(I(I-1))+S_{1}\right] \\
& +T_{1}\left(\lambda E(I) T_{2}\right)^{2} E\left(B_{2}^{2}\right)+2 \theta(\lambda E(I))^{2} T_{1}^{2} T_{2} E\left(B_{1}\right) E\left(V_{1}\right) \\
& +\theta E\left(V_{1}\right) T_{2} S_{1}+\theta E\left(V_{1}\right) T_{1} S_{2}+2 \theta T_{1} T_{2}^{2}(\lambda E(I))^{2} E\left(B_{2}\right) E\left(V_{1}\right) \\
& +\theta T_{1} T_{2} C_{1}+\theta p E\left(V_{2}\right)\left[2(\lambda E(I))^{2} T_{1}^{2} T_{2} E\left(B_{1}\right)+T_{2} S_{1}+T_{1} S_{2}\right] \\
& +\theta p T_{1} T_{2}\left[2(\lambda E(I))^{2} T_{2} E\left(B_{2}\right) E\left(V_{2}\right)+2(\lambda E(I))^{2} E\left(V_{1}\right) E\left(V_{2}\right)\right] \\
& +\theta p T_{1} T_{2} C_{2}+\alpha_{1} T_{1} E\left(B_{1}\right)\left[T_{2} D_{1}+S_{2} E\left(R_{1}\right)\right] \\
& +\alpha_{1} T_{2} E\left(R_{1}\right)\left[S_{1} E\left(B_{1}\right)+\left(\lambda E(I) T_{1}\right)^{2} E\left(B_{1}^{2}\right)\right] \\
& +\alpha_{2} E\left(R_{2}\right)\left[T_{2} E\left(B_{2}\right) S_{1}+T_{1}\left[S_{2} E\left(B_{2}\right)+\left(\lambda E(I) T_{2}\right)^{2} E\left(B_{2}^{2}\right)\right]\right] \\
& \left.+\alpha_{2} T_{1} T_{2} E\left(B_{2}\right)\left[2(\lambda E(I))^{2} T_{1} E\left(B_{1}\right) E\left(R_{2}\right)+D_{2}\right]\right) \\
& +\left(b(b-1)-2 \theta p(\lambda E(I))^{2} E\left(V_{1}\right) E\left(V_{2}\right)-\theta(1-p) C_{1}\right. \\
& -\theta p \lambda E(I(I-1))\left(E\left(V_{1}\right)+E\left(V_{2}\right)\right)-\theta p(\lambda E(I))^{2}\left(E\left(V_{1}^{2}\right)+E\left(V_{2}^{2}\right)\right) \\
& -2 \theta(\lambda E(I))^{2}\left(E\left(V_{1}\right)+p E\left(V_{2}\right)\right)\left(T_{1} E\left(B_{1}\right)+T_{2} E\left(B_{2}\right)\right) \\
& -2(\lambda E(I))^{2} T_{1} T_{2} E\left(B_{1}\right) E\left(B_{2}\right)-S_{1} E\left(B_{1}\right)-S_{2} E\left(B_{2}\right)
\end{aligned}
$$




$$
\begin{aligned}
& \left.-(\lambda E(I))^{2}\left[T_{1}^{2} E\left(B_{1}^{2}\right)+T_{2}^{2} E\left(B_{2}^{2}\right)\right]\right) \times\left(\lambda E(I) T_{1} T_{2} \sum_{r=0}^{a-1} Q_{r}\right) \\
& +\left(b-\lambda E(I)\left(A_{1}+\theta E\left(V_{1}\right)+\theta p E\left(V_{2}\right)\right)\right) \times\left[\sum_{r=0}^{a-1} Q_{r} T_{2}\left(2 T_{1} \lambda E(I(I-1))+\alpha_{1}(\lambda E(I))^{2} E\left(R_{1}^{2}\right)\right)\right. \\
& \left.\left.+2 \lambda E(I) T_{1} T_{2} \sum_{r=0}^{a-1} r Q_{r}+T_{1}\left(\lambda E(I(I-1)) T_{2}+\alpha_{2}(\lambda E(I))^{2} E\left(R_{2}^{2}\right)\right) \sum_{r=0}^{a-1} Q_{r}\right]\right]
\end{aligned}
$$

where

$$
\begin{aligned}
X_{1}= & \lambda \sum_{r=0}^{a-1} Q_{r}(E(I)+r-b)+\lambda \sum_{r=0}^{a-1} \sum_{n=1}^{b-r-1} c_{n} Q_{r}(b-n-r)+\sum_{r=0}^{b-1}(b-r) W_{r} \\
X_{2}= & \lambda \sum_{r=0}^{a-1} \sum_{n=1}^{b-r-1} c_{n} Q_{r}(b(b-1)-(n+r)(n+r-1)) \\
& +\lambda \sum_{r=0}^{a-1} Q_{r}(E(I(I-1))+2 E(I) r+r(r-1)-b(b-1)) \\
& +\sum_{r=0}^{b-1}(b(b-1)-r(r-1)) W_{r} \\
A_{1}= & T_{1} E\left(B_{1}\right)+T_{2} E\left(B_{2}\right), \quad T_{1}=1+\alpha_{1} E\left(R_{1}\right), \quad T_{2}=1+\alpha_{2} E\left(R_{1}\right) \\
S_{1}= & \lambda E(I(I-1)) T_{1}+\alpha_{1}(\lambda E(I))^{2} E\left(R_{1}^{2}\right) ; S_{2}=\lambda E(I(I-1)) T_{2}+\alpha_{2}(\lambda E(I))^{2} E\left(R_{2}^{2}\right) \\
C_{1}= & \lambda E(I(I-1)) E\left(V_{1}\right)+(\lambda E(I))^{2} E\left(V_{1}^{2}\right) ; C_{2}=\lambda E(I(I-1)) E\left(V_{2}\right)+(\lambda E(I))^{2} E\left(V_{2}^{2}\right) \\
D_{1}= & \lambda E(I(I-1)) E\left(R_{1}\right)+(\lambda E(I))^{2} E\left(R_{1}^{2}\right) ; D_{2}=\lambda E(I(I-1)) E\left(R_{2}\right)+(\lambda E(I))^{2} E\left(R_{2}^{2}\right) \\
E(I)= & C^{\prime}(1), \quad E(I(I-1))=C^{\prime \prime}(1)
\end{aligned}
$$

- The average time a customer spends in the queue $\left(W_{q}\right)$ are found by using the Little's formula,

$$
W_{q}=\frac{L_{q}}{\lambda E(I)}
$$

\section{QueUe Size Distribution at A DEPARTURE EPOCH}

In this section, we derive the probability generating function of the queue size distribution at a departure epoch of this model is given in the proof of Theorem 1

Theorem 1 Under the steady-state condition, the PGF of the queue size distribution at a departure epoch of this model is given by

$$
P^{+}(z)=\frac{\left(\begin{array}{c}
{\left[\lambda \sum_{r=0}^{a-1} Q_{r}\left(C(z) z^{r}-z^{b}\right)+\sum_{r=0}^{b-1}\left(z^{b}-z^{r}\right) W_{r}\right.} \\
\left.+\lambda \sum_{r=0}^{a-1} \sum_{n=1}^{b-r-1} c_{n} Q_{r}\left(z^{b}-z^{n+r}\right)\right] \times b(1-\rho) \\
\times((1-\theta)+\theta K(z))\left(\bar{B}_{1}\left(\psi_{1}(z)\right) \bar{B}_{2}\left(\psi_{2}(z)\right)\right)
\end{array}\right)}{\left(\left[z^{b}-((1-\theta)+\theta K(z))\left(\bar{B}_{1}\left(\psi_{1}(z)\right) \bar{B}_{2}\left(\psi_{2}(z)\right)\right)\right] X_{1}\right)} .
$$


Analysis of $M^{X} / \mathrm{G}(\mathrm{a}, \mathrm{b}) / 1$ Queueing System with Two Phases of Service Subject to Server Breakdown and Extended Bernoulli Vacations

Proof: Following the argument of PASTA (See Wolf [17]). We state that a departing customer will see ' $\mathrm{j}$ ' customer in the queue just after a departure if and only if there were ' $\mathrm{j}$ ' customer in the queue after completing the second phase of service or both the first type of Bernoulli vacation and second type of extended vacation just before the departure.

Let $P^{+}(z)=\sum_{j=0}^{\infty} p_{j}^{+} z^{j}$ be the probability that there are ' $\mathrm{j}$ ' customers in the queue at a departure epoch, we may write

$$
p_{j}^{+}=K_{0}(1-\theta) \int_{0}^{\infty} P_{2, j}(x) \mu_{2}(x) d x+K_{0}(1-p) \int_{0}^{\infty} V_{1, j}(x) \gamma_{1}(x) d x+K_{0} \int_{0}^{\infty} V_{2, j}(x) \gamma_{2}(x) d x, j \geq 0
$$

where $K_{0}$ is the normalizing constant.

By multiplying both sides of the equation (73) by $z^{j}$ summation over $\mathrm{j}$ from 0 to $\infty$, and use the equations (50),(51), and (52) (after applying the Tauberian property), we get on simplification

$$
P^{+}(z)=\frac{K_{0}\left(\begin{array}{c}
{\left[\lambda \sum_{r=0}^{a-1} Q_{r}\left(C(z) z^{r}-z^{b}\right)+\lambda \sum_{r=0}^{a-1} \sum_{n=1}^{b-r-1} c_{n} Q_{r}\left(z^{b}-z^{n+r}\right)\right.} \\
\left.+\sum_{r=0}^{b-1}\left(z^{b}-z^{r}\right) W_{r}\right] \\
\times((1-\theta)+\theta K((z)))\left(\bar{B}_{1}\left(\psi_{1}(z)\right) \bar{B}_{2}\left(\psi_{2}(z)\right)\right)
\end{array}\right)}{\left[z^{b}-((1-\theta)+\theta K(z))\left(\bar{B}_{1}\left(\psi_{1}(z)\right) \bar{B}_{2}\left(\psi_{2}(z)\right)\right)\right]} .
$$

From $P^{+}(1)=1, \quad$ we get

$$
K_{0}=\frac{b(1-\rho)}{X_{1}}
$$

where

$$
\rho=\frac{\left(\lambda E(I)\left(A_{1}+\theta E\left(V_{1}\right)+\theta p E\left(V_{2}\right)\right)\right)}{b}<1
$$

Inserting equation (75) into (74) we get the PGF of the queue size distribution at a departure epoch of this model.

Next the mean queue size of this model is given in corollary 1.

Corollary 1 Under the stability conditions, the mean number of customers in the queue at a departure epoch $L_{d}$ is given by

$$
\begin{aligned}
& L_{d}=b \rho+ \frac{X_{2}}{2 X_{1}}-\frac{b-1}{2(1-\rho)} \\
&(\lambda E(I))^{2}\left[2 \theta p E\left(V_{1}\right) E\left(V_{2}\right)+\theta\left(E\left(V_{1}^{2}\right)+p E\left(V_{2}^{2}\right)\right)\right. \\
&+2 \theta\left(E\left(V_{1}\right)+p E\left(V_{2}\right)\right)\left(T_{1} E\left(B_{1}\right)+T_{2} E\left(B_{2}\right)\right) \\
&+2 T_{1} T_{2} E\left(B_{1}\right) E\left(B_{2}\right)+\alpha_{1} E\left(B_{1}\right) E\left(R_{1}^{2}\right)+T_{1}^{2} E\left(B_{1}^{2}\right) \\
&+\left.+\alpha_{2} E\left(B_{2}\right) E\left(R_{2}^{2}\right)+T_{2}^{2} E\left(B_{2}^{2}\right)\right] \\
& 2 b(1-\rho)
\end{aligned}
$$


Analysis of $M^{X} / \mathrm{G}(\mathrm{a}, \mathrm{b}) / 1$ Queueing System with Two Phases of Service Subject to Server Breakdown and Extended Bernoulli Vacations

Proof: The result follows directly by differentiating (77) with respect to $\mathrm{z}$ and then taking limit $z \rightarrow 1$ by using the L'Hopital's rule.

Where $X_{1}, X_{2}, T_{1}, T_{2}, A_{1}, E(I), E(I(I-1))$ are given in Section 6 .

\section{Particular Cases}

Case 1: If batch arrival, single server providing single phase of service and no breakdown is considered, then equation (69) reduces to

$$
P(z)=\frac{Q\left[(1-\bar{B}(\phi(z)))+\theta \bar{B}(\phi(z))\left(1-\bar{V}_{1}(\phi(z))\right)+\theta p \bar{B}(\phi(z)) \bar{V}_{1}(\phi(z))\left(1-\bar{V}_{2}(\phi(z))\right)\right.}{((1-\theta)+\theta K(z, s)) \bar{B}(\phi(z))-z}
$$

where $Q=1-\rho, \phi(z)=\lambda(1-C(z)) \rho=\lambda E(I)\left(E(B)+\theta E\left(V_{1}\right)+\theta p E\left(V_{2}\right)\right)$

These expressions are exactly matched with the results by Ayyappan and Shymala (2013).

Case 2: If single arrival, single server, two phase service, no extended vacation is considered, then equation (69),(72) and (79) reduces to

$$
\begin{aligned}
& Q\left[\phi(z)\left(1-\bar{B}_{1}\left(\psi_{1}(z)\right)\right) \psi_{2}(z)+\phi(z) \psi_{1}(z) \bar{B}_{1}\left(\psi_{1}(z)\right)\left(1-\bar{B}_{2}\left(\psi_{2}(z)\right)\right)\right. \\
&+\theta \psi_{1}(z) \psi_{2}(z) \bar{B}_{1}\left(\psi_{1}(z)\right) \bar{B}_{2}\left(\psi_{2}(z)\right)\left(1-\bar{V}_{1}(\phi(z))\right) \\
&+\alpha_{1} \psi_{2}(z)\left(1-\bar{B}_{1}\left(\psi_{1}(z)\right)\right)\left(1-\bar{R}_{1}(\phi(z))\right) \\
& P(z)=\left.+\alpha_{2} \psi_{1}(z) \bar{B}_{1}\left(\psi_{1}(z)\right)\left(1-\bar{B}_{2}\left(\psi_{2}(z)\right)\right)\left(1-\bar{R}_{2}(\phi(z))\right)\right] \\
&\left(\left((1-\theta)+\theta \bar{V}_{1}(\phi(z))\right) \bar{B}_{1}\left(\psi_{1}(z)\right) \bar{B}_{2}\left(\psi_{2}(z)\right)-z\right) \psi_{1}(z) \psi_{2}(z)
\end{aligned}
$$

and

$$
\begin{aligned}
& P^{+}(z)=\frac{Q(1-z)\left((1-\theta)+\theta \bar{V}_{1}(\phi(z))\right) \bar{B}_{1}\left(\psi_{1}(z)\right) \bar{B}_{2}\left(\psi_{2}(z)\right)}{\left((1-\theta)+\theta \bar{V}_{1}(\phi(z))\right) \bar{B}_{1}\left(\psi_{1}(z)\right) \bar{B}_{2}\left(\psi_{2}(z)\right)-z} \\
& L_{d}=\rho+\frac{T_{1} T_{2} \lambda^{2} E\left(B_{1}\right) E\left(B_{2}\right)}{1-\rho}+\frac{\lambda^{2} \theta\left[2 E\left(V_{1}\right) E\left(B_{1}\right) T_{1}+2 E\left(V_{1}\right) E\left(B_{2}\right) T_{2}+E\left(V_{1}^{2}\right)\right]}{2(1-\rho)} \\
& +\frac{\left.\lambda^{2}\left[T_{1}^{2} E\left(B_{1}^{2}\right)+\alpha_{1} E\left(R_{1}^{2}\right) E\left(B_{1}\right)+T_{2}^{2} E\left(B_{2}^{2}\right)+\alpha_{2} E\left(R_{2}^{2}\right)\right) E\left(B_{2}\right)\right]}{2(1-\rho)} \\
& \text { where } Q=1-\rho, \rho=\lambda\left(A_{1}+\theta E\left(V_{1}\right)\right) \\
& \quad \phi(z)=\lambda(1-z) \text { and } \psi_{i}(z)=\phi(z)+\alpha_{i}\left(1-\bar{R}_{i}(\phi(z))\right), i=1,2
\end{aligned}
$$

These expressions agree with the results by Gautam Choudhury and Mitali Deka (2012).

\section{Numerical ReSUltS}

In this section, we present some numerical examples using MATLAB in order to illustrate the effect of various parameters in the system performance measures of our system where batch size distribution of the arrival is geometric with mean 2 and two phases of service time follow Erlang-2 distribution and two types of vacation time and two phases of repair time follow exponential distribution. We assume arbitrary values to the parameters such that the stability condition is satisfied. Tables 1 to 3 gives computed values of the utilization factor $(\rho)$, the mean queue $\operatorname{size}\left(L_{q}\right)$, mean waiting time in the queue $\left(W_{q}\right)$ in our queueing model. 
Analysis of $M^{X} / \mathrm{G}(\mathrm{a}, \mathrm{b}) / 1$ Queueing System with Two Phases of Service Subject to Server Breakdown and Extended Bernoulli Vacations

The table 1 clearly shows that the arrival rate $(\lambda)$ increases, the utilization factor $(\rho)$, the mean queue size $\left(L_{q}\right)$ and the mean waiting time in the queue $\left(W_{q}\right)$ are also increases for the value of $a=2, b=5, \theta=0.3, p_{1}=0.5, \mu_{1}=8, \mu_{2}=14, \gamma_{1}=7, \gamma_{2}=8, \alpha_{1}=1, \alpha_{2}=1.1, \beta_{1}=2$, $\beta_{2}=2.5$.

Table 2 shows that the first phase of service rate increases, the utilization factor $(\rho)$, the mean queue size $\left(L_{q}\right)$ and the mean waiting time in the queue $\left(W_{q}\right)$ are decreases for the value of $a=2, b=5$, $\theta=0.3, p_{1}=0.5, \lambda=1, \mu_{2}=14, \gamma_{1}=7, \gamma_{2}=8, \alpha_{1}=1, \alpha_{2}=1.1, \beta_{1}=2, \beta_{2}=2.5$.

Table 3 shows that the second phase of service rate increases, the utilization factor $(\rho)$, the mean queue size $\left(L_{q}\right)$ and the mean waiting time in the queue $\left(W_{q}\right)$ are decreases for the value of $a=2$, $b=5, \theta=0.3, p_{1}=0.5, \lambda=1, \mu_{1}=8, \gamma_{1}=7, \gamma_{2}=8, \alpha_{1}=1, \alpha_{2}=1.1, \beta_{1}=2, \beta_{2}=2.5$.

Table 1. The effect of arrival rate $(\lambda)$ on $\rho, L_{q}, W_{q}$

\begin{tabular}{|c|c|c|c|}
\hline$\lambda$ & $\rho$ & $L_{q}$ & $W_{q}$ \\
\hline 1.00 & 0.1408 & 3.8415 & 1.9207 \\
\hline 1.25 & 0.1760 & 4.9276 & 1.9710 \\
\hline 1.50 & 0.2112 & 6.3085 & 2.1028 \\
\hline 1.75 & 0.2464 & 8.0492 & 2.2998 \\
\hline 2.00 & 0.2816 & 10.2889 & 2.5585 \\
\hline 2.25 & 0.3168 & 12.9732 & 2.8829 \\
\hline 2.50 & 0.3520 & 16.4129 & 3.2826 \\
\hline 2.75 & 0.3872 & 20.7468 & 3.7721 \\
\hline 3.00 & 0.4224 & 26.2359 & 4.3726 \\
\hline
\end{tabular}

Table 2. The effect of service rate $\left(\mu_{1}\right)$ on $\rho, L_{q}, W_{q}$

\begin{tabular}{|c|l|l|l|}
\hline$\mu_{1}$ & $\rho$ & $L_{q}$ & $W_{q}$ \\
\hline 3 & 0.2658 & 9.1982 & 4.5991 \\
\hline 4 & 0.2158 & 6.4896 & 3.2448 \\
\hline 5 & 0.1858 & 5.2564 & 2.6282 \\
\hline 6 & 0.1658 & 4.5688 & 2.2844 \\
\hline 7 & 0.1515 & 4.1362 & 2.0681 \\
\hline 8 & 0.1408 & 3.8415 & 1.9207 \\
\hline 9 & 0.1325 & 3.6309 & 1.8155 \\
\hline 10 & 0.1258 & 3.4745 & 1.7373 \\
\hline 11 & 0.1203 & 3.3526 & 1.6763 \\
\hline 12 & 0.1158 & 3.2551 & 1.6275 \\
\hline
\end{tabular}

Table 3. The effect of service rate $\left(\mu_{2}\right)$ on $\rho, L_{q}, W_{q}$

\begin{tabular}{|c|c|c|c|}
\hline$\mu_{2}$ & $\rho$ & $L_{q}$ & $W_{q}$ \\
\hline 3 & 0.2916 & 10.1454 & 5.0727 \\
\hline 4 & 0.2436 & 7.3645 & 3.6822 \\
\hline 5 & 0.2148 & 6.0918 & 3.0459 \\
\hline 6 & 0.1956 & 5.3792 & 2.6896 \\
\hline 7 & 0.1819 & 4.9296 & 2.4648 \\
\hline 8 & 0.1716 & 4.6223 & 2.3112 \\
\hline 9 & 0.1636 & 4.4002 & 2.2001 \\
\hline 10 & 0.1572 & 4.2327 & 2.1163 \\
\hline 11 & 0.1520 & 4.1021 & 2.0511 \\
\hline 12 & 0.1476 & 3.9977 & 1.9989 \\
\hline
\end{tabular}


Analysis of $M^{X} / \mathrm{G}(\mathrm{a}, \mathrm{b}) / 1$ Queueing System with Two Phases of Service Subject to Server Breakdown and Extended Bernoulli Vacations

In Figure 1 shows that the utilization factor $(\rho)$, the average queue length $\left(L_{q}\right)$ and average waiting time in the queue $\left(W_{q}\right)$ increases for the increasing values of the arrival rate $\lambda$.

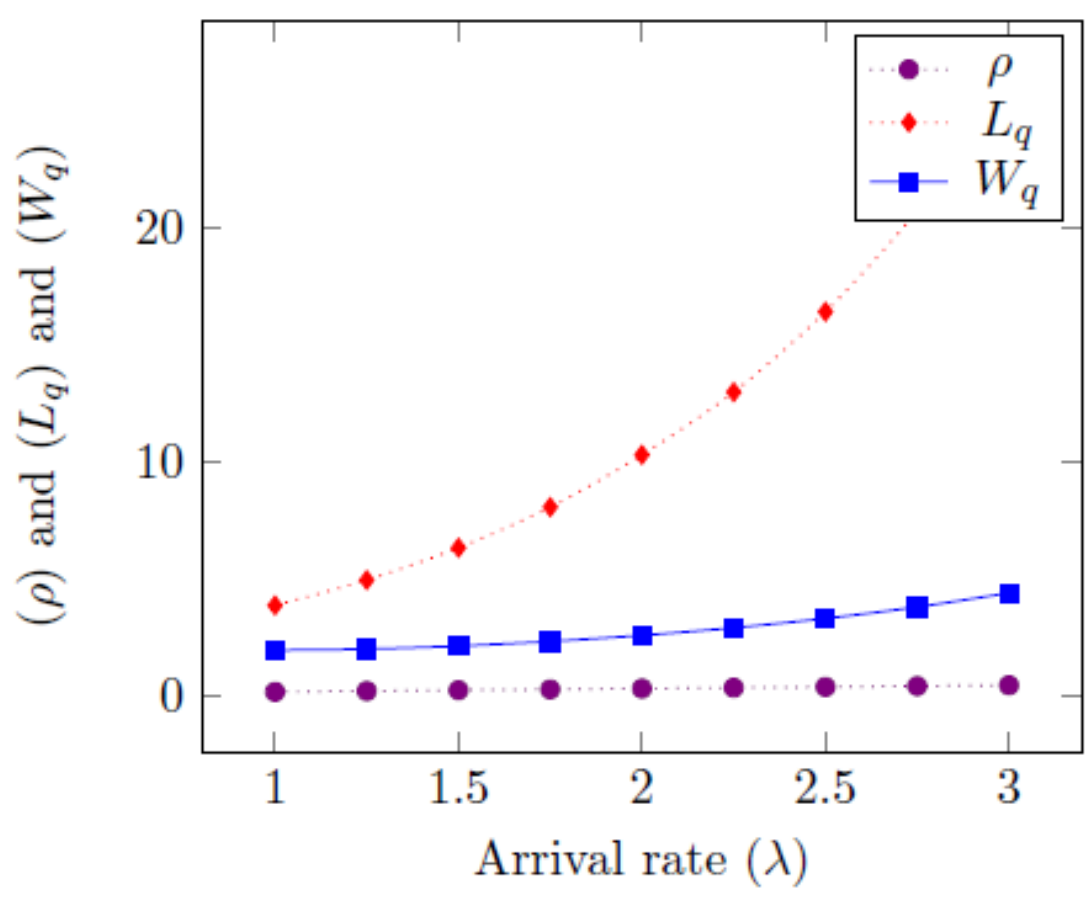

Figure 1: $\rho, L_{q}, W_{q}$ verses arrival rate $\lambda$

Similarly, In Figure 2 and 3 shows that the utilization factor $(\rho)$, the average queue length $\left(L_{q}\right)$ and average waiting time in the queue $\left(W_{q}\right)$ decreases for the increasing values of service rates $\mu_{1}$ and $\mu_{2}$.

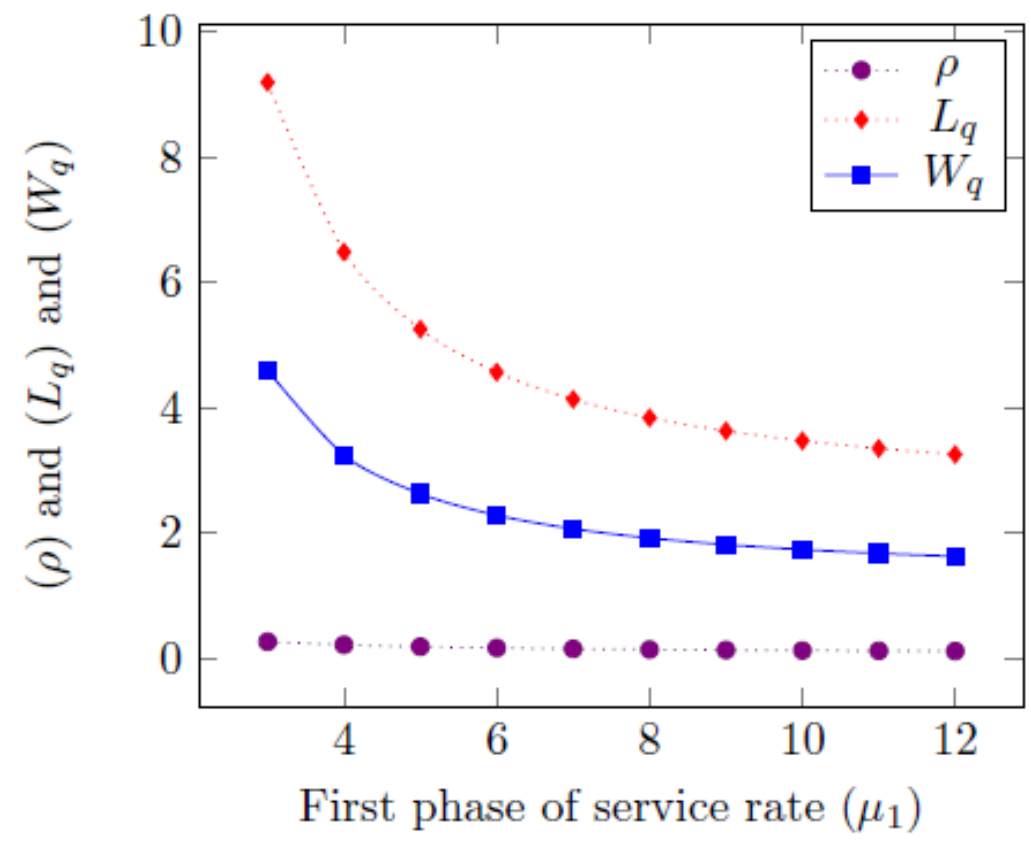

Figure 2: $\rho, L_{q}, W_{q}$ verses First phase of service rate $\mu_{1}$ 


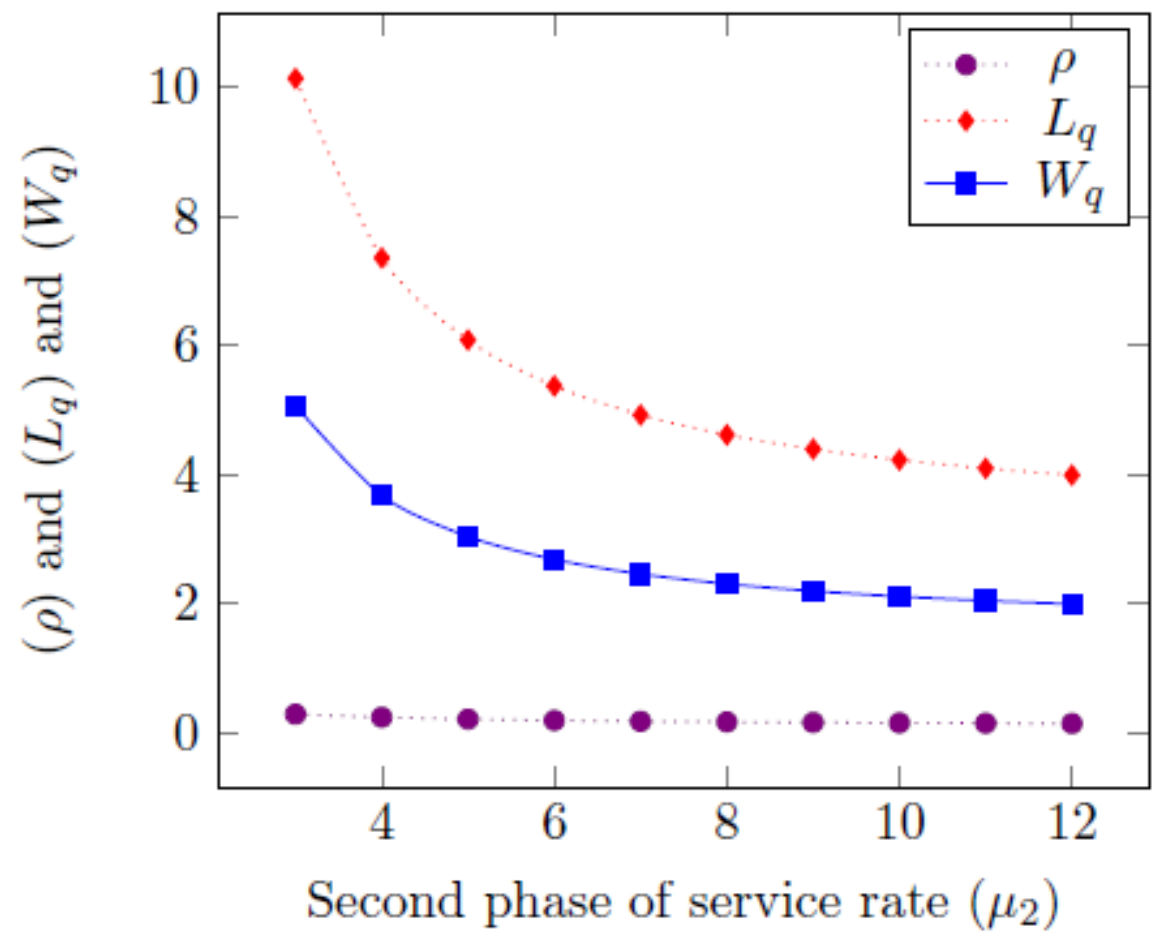

\section{Figure 3: $\rho, L_{q}, W_{q}$ verses Second phase of service rate $\mu_{2}$}

\section{CONCLUSION}

In this paper, we have studied an $M^{X} / \mathrm{G}(\mathrm{a}, \mathrm{b}) / 1$ queueing system with two phases of service subject to server breakdown and extended Bernoulli vacation. We derive the probability generating function of the number of customers in the queue at a random epoch in transient and steady state conditions and also we obtained the queue size distribution at a departure epoch under the steady state conditions. The performance measures of the system state probabilities, the mean queue size and the average waiting time in the queue are found by using the supplementary variable technique. Some particular cases are determined. Finally, the results are validated with the help of numerical illustrations.

\section{REFERENCES}

[1] Norman Bailey, T.J., On queueing processes with bulk service, J.Roy. Statist. Soc. Ser., 16, 80-87, (1954).

[2] Neuts, M.F., A general class of bulk queues with poisson input, The Annals of Mathematical Statistics, 38, 759-770, (1967).

[3] Holman, D.F., Chaudhry, M.L and Ghosal, A.,Some results for the general bulk service queueing system, Bull. Austral. Math. Soc., 23, 161-179, (1981).

[4] Chaudhry, M.L. and Templeton, J.G.C., A First Course in Bulk Queues, John Wiley Sons, USA, (1983).

[5] Banerjee, A., Gupta, U.C. and Sikdar, K. , Analysis of finite-buffer bulk-arrival bulk service queue with variable service capacity and batch-size-dependent service, International Journal of Mathematics in Operational Research,5(3), 358-386,(2013).

[6] Sikdar, K. and Samanta, S. K., Analysis of a finite buffer variable batch service queue with batch Markovian arrival process and servers vacation, OPSEARCH, 53( 3), 553-583, (2016).

[7] Jeyakumar, S. and Senthilnathan, B., Steady state analysis of bulk arrival and bulk service queueing model with multiple working vacations, International Journal of Mathematics in Operational Research, 9(3), 375-394, (2016)

[8] Choudhury, G. and Deka, M., A batch arrival unreliable Bernoulli vacation model with two phases of service and general retrial times, International Journal of Mathematics in Operational Research, 7( 3), 318-347, . (2015)

[9] Badamchi Zadeh, Shahkar, A. G. H., A Two Phases Queue System with Bernoulli Feedback and Bernoulli Schedule Server Vacation,Information and Management Sciences 19(2),329-338, (2008). 
Analysis of $M^{X} / \mathrm{G}(\mathrm{a}, \mathrm{b}) / 1$ Queueing System with Two Phases of Service Subject to Server Breakdown and Extended Bernoulli Vacations

[10] Haridass, M., Arumuganathan, R. and Senthilkumar, M., Cost analysis of a bulk service retrial queue, International Journal of Operational Research, 14(1), 94-119,(2012).

[11] Choudhury, G., A two-phase batch arrival retrial queueing system with Bernoulli vacation schedule, Applied Mathematics and Computation, 188, 2, 1455-1466, (2007).

[12] Madan, K. C., Gautam Choudhury., A Single Server Queue with Two Phases of Heterogeneous Service under Bernoulli Schedule and a General Vacation Time, Information and Management Sciences, 16(2), 116 (2005).

[13] Ayyappan, G. and Shymala, S., Time Dependent Solution of Batch Arrival Queueing System with Random Breakdowns and Second Optional Server Vacation, International Journal of Computational and Applied Mathematics, 8(1), 71-86, (2013).

[14] Al-Khedhairi, A. and Tadj, L., A bulk service queue with a choice of service and reservice under Bernoulli schedule, International Journal of Contemporary Mathematical Sciences, 2(23), 1107-1120, (2007).

[15] Rajadurai, P., Varalakshmi, M., Saravanarajan, M.C., and Chandrasekaran, V.M. , Analysis of $M^{X} / G / 1$ retrial queue with two phase service under Bernoulli vacation schedule and random breakdown, Int. J. Mathematics in Operational Research, 7, 19-41, (2015).

[16] Choudhury, G. and Deka, M. , A single server queueing system with two phases of service subject to server breakdown and Bernoulli vacation, Applied Mathematical Modelling, 36(12), 6050-6060, (2012).

[17] Wolf, R.W., Poisson arrivals see time averages, Operational Research, 30, 223-231, (1982).

AUTHORS' BIOGRAPHY

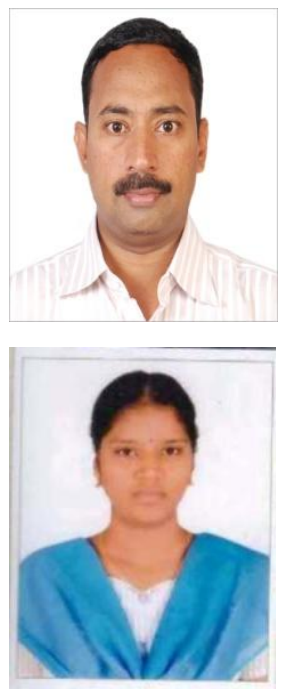

Dr. G.Ayyappan is a professor and Head of Mathematics Department,in Pondicherry Engineering College. He has completed his Ph.D in Annamalai University and having 23 years of teaching experience. He has published more than 100 papers in reputed International journals and published more than 30 paers in National and International conferences. His areas of interests are Queueing theory,Reliability Analysis and Inventory models.

R. Supraja has completed Master of Science degree in Mathematics from Pondicherry University, Puducherry. At present, she is a Research Scholar in Department of Mathematics, Pondicherry Engineering College. Her area of interests are stochastic processes and queueing theory.

Citation: G. Ayyappan, R. Supraja, "Analysis of $M^{X} / G(a, b) / 1$ Queueing System with Two Phases of Service Subject to Server Breakdown and Extended Bernoulli Vacations ", International Journal of Scientific and Innovative Mathematical Research, vol. 5, no. 11, p. 32-51, 2017., http://dx.doi.org/10.20431/23473142.0511004

Copyright: () 2017 Authors. This is an open-access article distributed under the terms of the Creative Commons Attribution License, which permits unrestricted use, distribution, and reproduction in any medium, provided the original author and source are credited. 